\title{
Device-Dependent Readability for Improved Text Understanding
}

\author{
A-Yeong Kim \\ Hyun-Je Song \\ Seong-Bae Park \\ Sang-Jo Lee \\ School of Computer Science and Engineering \\ Kyungpook National University \\ Daegu, 702-701, Korea \\ \{aykim,hjsong, sbpark\}@sejong.knu.ac.kr, sjlee@knu.ac.kr
}

\begin{abstract}
Readability is used to provide users with highquality service in text recommendation or text visualization. With the increasing use of handheld devices, reading device is regarded as an important factor for readability. Therefore, this paper investigates the relationship between readability and reading devices such as a smart phone, a tablet, and paper. We suggest readability factors that are strongly related with the readability of a specific device by showing the correlations between various factors in each device and human-rated readability. Our experimental results show that each device has its own readability characteristics, and thus different weights should be imposed on readability factors according to the device type. In order to prove the usefulness of the results, we apply the device-dependent readability to news article recommendation.
\end{abstract}

\section{Introduction}

Readability is a function that maps a given text into a readability score by considering "how easily the text is read and understood" (Richards et al., 1992; Zamanian and Heydari, 2012). Normally, the readability score is formulated as a combination of various factors. These factors reflect the easiness and understanding of the text and include text presentation format, font size, average ratio of annotated images, and sentence length (Hasegawa et al., 2008; Kitson, 1927; Ma et al., 2012; Öquist, 2006). Therefore, readability can be used to provide satisfiable services in text recommendation or text visualization.

The study on readability has begun in the education field to measure the level of a text. With the success of using readability in education (François and Fairon, 2012; Heilman et al., 2008; Ma et al., 2012), readability has been used in a range of domains recently. For example, in document retrieval, readability is used to provide documents to non-expert users so that they can read the retrieved documents easily (Jameel et al., 2012; Yan et al., 2006). In text mining, readability has been employed to analyze the characteristics of text. Especially, Hillbom showed the differences in readabil- ity between broadsheet newspapers and tabloids that share a similar political stance (Hillbom, 2009).

There is one important issue of readability that has not been studied in natural language processing. It is a reading device. That is, previous studies focused only on text printed on paper. However, with the increasing use of hand-held devices, people in these days use various reading devices such as a tablet and a smart phone as well as a paper. Readability score can be different according to the device type, because each device has its own idiosyncrasy. For example, assume that a system recommends the same news article to both user A who reads it in her smart phone and user B who reads it on paper. Although both users read the same article, user A might believe that her article is more difficult to read than user $B$ because of the screen size of her smart phone.

This paper explores the relationship between reading devices and readability. For this purpose, we first investigate whether readability changes according to device type or not. Then, we analyze which readability factors are affected by reading devices. To see the relationship between readability factors and devices, various well-known readability factors are computed for news articles collected from an Internet portal. At the same time, the readability of each article is also manually rated. When the readability is rated manually, it is done three times for different reading devices of a smart phone, a tablet, and paper. The factors that affect the readability actually in each device are found out through the correlations between the factors and the manually-labeled readability. Some factors are important to the readability of smart phone, but insignificant to that of paper. Therefore, we discover the importance of each readability factor for each device by analyzing the correlations.

The usefulness of the device-dependent readability is proven by applying it to news article recommendation. That is, different importance weights for readability factors are considered according to device type when recommending news articles. Our experimental results show that the performance of news article recommendation gets best when the device used for reading news articles is identical to the device used for measuring readability. Therefore, it is essential to consider different importance weights according to device type 
in news article recommendation. It also proves that the proposed device-dependent readability reflects the characteristics of reading devices well.

The rest of this paper is organized as follows. We first review related studies on readability. Next, we introduce various readability factors and propose the device-dependent readability. Then, the news article recommendation using the device-dependent readability is explained. This recommendation is prepared to prove the usefulness of the device-dependent readability. In the experiments, we present the experimental results on the relationship between reading devices and readability. We also describe the experiments on news recommendation using the device-dependent readability and present their results. Finally, we summarize our research.

\section{Related work}

The history of readability studies began in the $1800 \mathrm{~s}$. Early studies focused on the frequency of easy words, sentence length, and word length (Huldén, 2004). Flesch designed a formula to calculate "reading ease" using only the average word length and sentence length (Flesch, 1948). He adjusted the relative importance between word length and sentence length using 100 words selected randomly from a corpus. This formula is called the Flesch-Kincaid formula, and is generally used in measuring the readability of a textbook (Kincaid et al., 1975). Dale and Chall (1949) defined a list of 3,000 easy words. Then, they used the average sentence length and the percentage of words not included in the list. These studies simply used superficial factors, and thus do not reflect syntactic factors.

Recent studies on readability use various factors including syntactic ones, and combine them to produce a highly predictive model of readability. François and Faircon (2012) proposed a readability formula with 46 textual factors for French as a foreign language. The factors represent lexical, syntactic, and semantic characteristics of sentences, and the specificities of French. They are extracted from 28 French Foreign Language (FFL) textbooks written for adults learning FFL. On the other hand, Pitler and Nenkova (2008) showed the relation between readability factors and readability. They used human ratings from the Wall Street Journal corpus, and computed the correlations between the readability factors and the average human ratings. According to their results, the average number of verb phrases in a sentence, the number of words in an article, the likelihood of the vocabulary, and the likelihood of the discourse relations are highly correlated with human ratings. However, these studies did not consider the reading devices, but focused on how well a text is written. Since the readability can be differentiated according to reading device, a reading device should be considered when computing the readability of a given text.

To the best of our knowledge, there are few studies on the readability on mobile devices that do not con- sider language-related aspects. Most studies on mobile devices focused on the development of new text format and layout to help users read documents easily. Öquist (2006) proposed a new text presentation format called the dynamic Rapid Serial Visual Presentation. According to his experimental results, this format helps to reduce eye movements. On the other hand, Hasegawa et al. (2008) evaluated the readability of documents on mobile devices with regard to screen and font size. They reported that the readability is improved when the characters are vertically enlarged. Readability on mobile devices is not reflected only by the visualization factors, but also by textual factors. Therefore, this paper explores the readability factors that reflect the lexical and grammatical complexity of text and are affected by reading devices.

\section{Readability Factors}

Table 1 lists the readability factors used in this paper. Basically, they are based on the factors proposed by Pitler and Nenkova (2008). However, some factors are excluded and some new factors are added. This is because some of their factors are computationally infeasible and language-dependent. As a result, we have thirteen readability factors. These readability factors are divided into four types: superficial, lexical, syntactic factors, and lexical cohesion.

\subsection{Superficial Factors}

Superficial factors were used in most early readability studies (Dale and Chall, 1949; Flesch, 1948; Kincaid et al., 1975), and reflect the construction of a text. We investigate four factors: text length (TL), sentence length (SL), average number of words per sentence (WS), and average number of characters per word (CW). Since longer text is perceived as "harder-to-read" than short one, these factors are all reciprocally related with readability.

The first two factors are related to length. TL counts the number of characters in a text, whereas SL computes the number of sentences. When a writer attempts to write many topics in a text, she tends to use many kinds of words simultaneously. As a result, the text becomes longer and more complex. Such long length of text disturbs a reader's comprehension of the text, and then it is more difficult for the reader to read the text (Heilman et al., 2008).

WS counts the average number of words per sentence, and CW reflects the average number of characters per word. When they are large, the sentence is difficult to read, which leads to difficulties in understanding the text. Especially, CW reflects compound nouns and technical words. For instance, compound nouns in Korean are usually long, because there is no spacing between words in a compound noun. For example, let us consider a compound noun, "Daehanmingukjungboo," which means the Korean government. Actually this compound noun consists of two independent nouns. 


\begin{tabular}{|c|c|c|}
\hline Type of Factors & Abbr. & Description \\
\hline \multirow{4}{*}{ Superficial factors } & TL & The number of characters in a text \\
\hline & SL & The number of sentences in a text \\
\hline & WS & Average number of words per sentence \\
\hline & $\mathrm{CW}$ & Average number of characters per word \\
\hline Lexical factor & LL & Article likelihood estimated by language model \\
\hline \multirow{4}{*}{ Syntactic factors } & PTD & Average parse tree depths per sentence \\
\hline & $\mathrm{NP}$ & Average number of noun phrases per sentence \\
\hline & $\mathrm{VP}$ & Average number of verb phrases per sentence \\
\hline & SBAR & Average number of subordinate clauses per sentence \\
\hline \multirow{4}{*}{ Lexical cohesion } & $\cos$ & Average cosine similarity between pairs of adjacent sentences \\
\hline & WO & Average word overlap between pairs of adjacent sentences \\
\hline & NPO & Average word overlap over noun and pronoun only \\
\hline & $\mathrm{PRP}$ & Average number of pronouns per sentence \\
\hline
\end{tabular}

Table 1: Description of readability factors

One is "Daehanminguk" meaning Korea and the other is "Jungboo" meaning a government. The two are concatenated to form a compound noun and become a long single word. In addition, many difficult words such as domain-specific terms tend to be long. Such lengthy words make it difficult to read a text.

\subsection{Lexical Factor}

Lexical factor determines whether a given text consists of frequent words. Texts that express a new trend in various fields often use many newly coined words. Such neologisms make it difficult to read and understand a text. Therefore, an easily-understandable text is composed of widely-used words rather than unusual words.

In order to compute the use of frequent words in a text, a unigram language model is used as in the work of Pitler and Nenkova (2008). In this model, the log likelihood of text $t$ is computed by

$$
\sum_{w \in t} C(w) \cdot \log P(w \mid B)
$$

where $P(w \mid B)$ is the probability of a word $w$ according to a background corpus $B$, and $C(w)$ is the number of times that $w$ appears in $t$.

This factor examines the familiarity of the words used in the text. The more frequently a word appears in the background corpus, the more familiar it is regarded. The frequency of a word $w$ is then reflected into $P(w \mid B)$ computed from the independent background corpus $B$. Therefore, the factor $\mathrm{LL}$ is positively related with readability.

\subsection{Syntactic Factors}

Syntactic factors reflect sentence complexity directly that affects human processing of a sentence. We consider the average parse tree depth per sentence (PTD), the average number of noun phrases per sentence (NP), the average number of verb phrases per sentence (VP), and the average number of subordinate clauses per sen- tence (SBAR) as syntactic factors. These four factors were defined by Schwarm and Ostendorf (2005).

A reader regards a text as difficult when the sentences in the text have large parse tree depths or many subordinate clauses. Thus, PTD and SBAR are related negatively with readability. On the other hand, the relationship of NP and VP to readability are not one way. The large number of noun phrases in a text requires a reader to remember more items (Barzilay and Lapata, 2008; Pitler and Nenkova, 2008). However, it also makes the text more interesting. The texts written for adults actually contain more entities than those written for children (Barzilay and Lapata, 2008). The same is true for VP. The large number of verb phrases in a sentence makes the sentence more complex. However, people feel that a text is more easier to comprehend when related clauses are grouped together (Bailin and Grafstein, 2001).

\subsection{Lexical Cohesion}

Lexical cohesion denotes how the sentences in a text are semantically connected. People usually bring continuous sentences into their mind at the same time, and interpret them as a single unit (Okazaki et al., 2005). In other words, a reader prefers text whose sentences are smoothly connected to text whose sentences are independent of one another. Therefore, sentence continuity plays a primary role in understanding an entire text.

In the classic study of cohesion, various uses of cohesive elements such as pronouns, definite articles, and topic continuity have been discussed (Halliday and Hasan, 1976). This paper uses the average cosine similarity (COS), word overlap (WO), word overlap over just nouns and pronouns (NPO) between pairs of adjacent sentences, and the average number of pronouns per sentence (PRP). COS, WO, and NPO are superficial measures of topic continuity, whereas $\mathrm{PRP}$ is an indicative feature of sentence continuity. High values for these factors imply that the sentences in the text are related somehow. Therefore, these factors are believed to be related positively with readability. 


\subsection{Measurement of Readability}

When a reading device $d$ is given, the readability of text $t$, represented as $R(t \mid d)$, is formulated as a combination of readability factors with their corresponding weight in the device. We assume that $w_{i \mid d}$, the weight of a readability factor $f_{i}$, is dependent on the reading device $d$. Following the previous work of Pitler and Nenkova (2008), we also assume that each readability factor affects readability independently. Therefore, readability is calculated as a weighted linear sum of all readability factors. That is, $R(t \mid d)$ is computed by

$$
R(t \mid d)=\sum_{i \in\{1,2, \ldots, M\}} w_{i \mid d} \cdot f_{i}(t)
$$

where $M$ is the number of readability factors.

Each weight $w_{i \mid d}$ is determined from a set of news articles $T$. We collected a large number of news articles from an Internet news portal. The readability of each article was manually labeled. This is done three times, since we have three different devices of a smart phone, a tablet, and paper. Since human rating of each article $t \in T$ is available for each device, $w_{i \mid d}$ 's can be estimated by linear regression. These weights are different according to the devices.

\section{News Article Recommendation by Device-Dependent Readability}

The fact that the weights $w_{i \mid d}$ in Equation (2) are different for each device $d$ implies that the readability measurement should be different depending on the device type. In order to see the usefulness of this devicedependent readability, we apply it to news article recommendation. News article recommendation aims to provide a user with news articles that interest the user. Thus, it selects a few articles that meet user preference from a gigantic amount of news events. Various methods have reported notable results in news article recommendation (Das et al., 2007; Li et al., 2010; Liu et al., 2010). In addition, with the recent interest in handheld devices, the demand for news recommendation on hand-held devices is increasing. However, there has been, at least as far as we know, no study on the readability of hand-held devices.

Device-dependent readability is reflected into news article recommendation through a re-ranking framework. Figure 1 depicts the overall process of suggesting news articles for a specific device with the devicedependent readability. The point of this figure is to measure how appropriate a news article is for a specific reading device. For this, a news recommendation system first chooses a set of news articles from a news repository based on its own criterion. Then, we re-rank them by the device-dependent readability to obtain the final set of ranked news articles for the device.

Formally, a news article recommendation ranks a set of articles, $\mathbf{A}=\left\{a_{1}, a_{2}, \ldots, a_{m}\right\}$, where $a_{i}$ represents the $i$-th article. The order between ranks $a_{1} \succ a_{2} \succ$

\begin{tabular}{|c|c|c|c|}
\hline & Min & Max & Average \\
\hline Article length & 68 & 610 & 346.5 \\
\# of sentences & 1 & 14 & 6.24 \\
\# of words per sentence & 8 & 33 & 16.93 \\
\# of words per article & 17 & 178 & 99.34 \\
\hline
\end{tabular}

Table 2: Statistics of the news article data

$\ldots \succ a_{m}$ should be satisfied by the criterion of the recommendation system. That is, assuming that the system has a score function $\operatorname{score}\left(a_{i}\right)$, score $\left(a_{i}\right)>$ score $\left(a_{j}\right)$ has to be met if $a_{i} \succ a_{j}$. Then, the top $k(k \leq m)$ articles of $\mathbf{A}$ by the score function are suggested as appropriate news articles. After that, the selected articles are re-ranked by another criterion, the device-dependent readability. That is, the final rank of an article within the selected set is determined by another function, rerank. Since this function has to reflect the device-dependent readability, it takes two parameters. One is an article, and the other is a device type. The re-rank function is modeled as

$$
\begin{aligned}
\operatorname{rerank}(a, d) & =R(a \mid d) \\
& =\sum_{i \in\{1,2, \ldots, M\}} w_{i \mid d} \cdot f_{i}(a) .
\end{aligned}
$$

As a result, the readability-based re-ranking module suggests the news articles based on how easily the articles are read on a specific reading device. Note that even the same article would be ranked differently according to the device type because the article is reranked by the device-dependent readability. At last, the top $k^{*}\left(k^{*} \leq k\right)$ re-ranked articles among them are suggested as final news articles.

\section{Experiments}

\subsection{Experiments on Readability Factors}

\subsubsection{Experiment Settings}

For the experiments of analyzing relationship between readability factors and readability, we collected a Korean news corpus from Naver News ${ }^{1}$. This corpus contains news articles from June 10, 2013 to June 25, 2013. We selected 74 articles randomly from the corpus which were used for readability formula and showing the relationships between readability factors. All selected articles belong to one of three categories: 'Politics', 'Entertainment', and 'Sports'. A set of these 74 news articles becomes $T$, and is used to compute the weights in Equation (2). Table 2 describes a simple statistics of the selected news articles. The shortest article consists of 68 characters, whereas the longest one has 610 characters. The average length of article is 346.5. The shortest article is written in one sentence, and the longest has 14 sentences. One article has approximately 6.24 sentences on average. In addition, the

\footnotetext{
${ }^{1} \mathrm{~A}$ Korean news portal of which web address is http: //news.naver.com.
} 


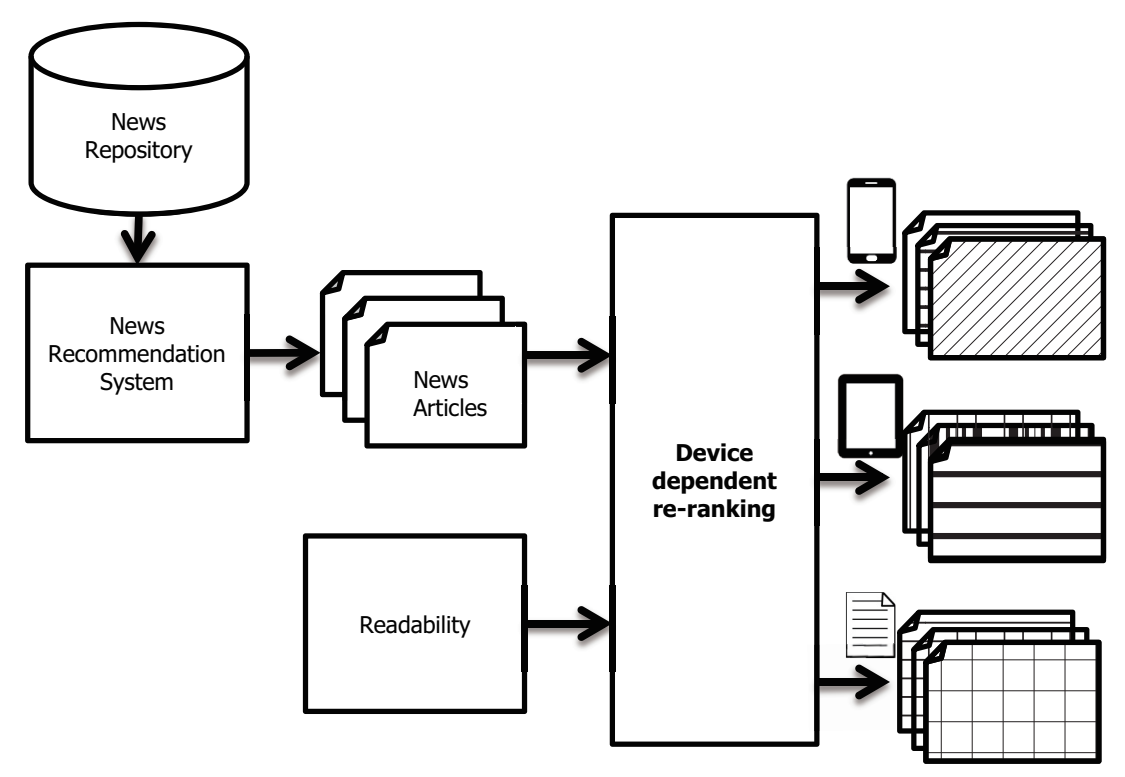

Figure 1: Overall process of re-ranking news articles based on device-dependent readability

number of words per sentence ranges from 8 to 33 , and the average is 16.93 . The minimum number of words in an article is 17, and the maximum number of words is 178 . An article is composed of 99.34 words on average.

In order to compute the lexical factor LL by Equation (1), a background corpus $B$ is required. Since this corpus should be independent from the news articles explained above, the Naver News is adopted again to generate $B$. For the background corpus $B$, we collected news articles from January 1, 2013 to September 6,2013 , but excluded the articles from June 10 to June 25 , because they are already used. This corpus consists of 298,729 articles with 3,264,104 distinct words.

The readability score for each article was manually labeled by three undergraduate students. To investigate the relationship between reading devices and readability, each article was read using three different reading devices. The Galaxy Note 1 with a 5 -inch screen is used as the smart phone, Galaxy Tab 10.1 with a 10.1inch screen is used as the tablet, and A4-size paper is used for the paper. That is, the human annotators read and rated 74 articles per device. The order of the devices where the annotators evaluated readability is smart phone, tablet, and paper. This order was maintained for all the experiments. All aspects but content texts were under control. For instance, font $=$ "Gothic, 12 pt" (this is most commonly used font and size that most Korean web pages and textbooks use), font color = "black", alignment = "both" were used for all three devices. In addition, the non-content aspects were exactly same for devices because the annotators of readability and the recommended articles shared the reading devices. Although these aspects affect readability and many previous studies already proved it, it is not our concern. We only attempt to capture how read-

\begin{tabular}{|c|c|c|c|}
\hline Reading device & Min & Max & Average \\
\hline Smart phone & 1.67 & 5 & $3.423 \pm 0.741$ \\
Tablet & 1.33 & 5 & $3.531 \pm 0.837$ \\
Paper & 2 & 5 & $3.360 \pm 0.594$ \\
\hline
\end{tabular}

Table 3: Readability scores given by human annotators

ability is affected by the content in different types of devices.

Human annotators can remember the content of news articles when they read articles with three devices. The human annotators were asked to read and evaluate many articles within a relatively short period. Therefore, before the main experiments, we performed a pilot experiment on the memory effects of previously read articles and verified it empirically. We hired three undergraduate students who were not involved in our main experiments. The students read the same 250 articles four times, and these also come from Naver News corpus which are not included the previous 74 articles. After their first reading, they read the articles again in 3, 7, and 14 days later. After 3 days, two students remembered the articles somewhat, but one student remembered them vaguely. Since they almost forgot the articles after 7 days, we placed 7 days interval between devices.

The readability score of an article was rated by the annotators using the questions in the work of Pitler and Nenkova (2008). We use only two of the questions, while they used four questions for the annotators. Their questions are intended to measure the extent of how well a text is written, how it fits together, how easy it is to understand, and how interesting it is. We can consider "well-written" and "fit-together" as a syntactic perspective, whereas "easy to understand" and "interesting" belong to a content perspective. For such a 


\begin{tabular}{|c|c|c|c|c|c|}
\hline \multicolumn{2}{|c|}{ Smart phone } & \multicolumn{2}{c|}{ Tablet } & \multicolumn{2}{c|}{ Paper } \\
\hline Factor & Value & Factor & Value & Factor & Value \\
\hline SL & -0.394 & SL & -0.370 & NP & 0.298 \\
TL & -0.293 & WS & 0.321 & WS & 0.278 \\
WS & 0.288 & LL & 0.253 & LL & 0.268 \\
LL & 0.249 & NP & 0.240 & VP & 0.244 \\
\hline
\end{tabular}

Table 4: Pearson correlation coefficients of important readability factors

reason, four questions can be summarized in two questions. The two questions used are

- How well-written is this article?

- How interesting is this article?

For these two questions, each annotator assigns a score between 1 and 5 to each article. Here, 1 point means that the article is worst and 5 point implies that it is best. A readability score of one human annotator is composed with the average of two questions (wellwritten, interesting). We used the average of three human annotators' readability scores in our experiments. Table 3 shows the readability scores of the articles for each device. According to this table, the readability score ranges from 1.67 to 5 for the smart phone, 1.33 to 5 for the tablet, and ranges from 2 to 5 for the paper. The average readability is 3.423 for the smart phone, 3.531 for the tablet, and 3.360 for the paper. To see the inter-judge agreement among annotators, the Kappa coefficient (Fleiss, 1971) is used. The Kappa values for the 'smart phone', 'tablet', and 'paper' are 0.342, 0.333 , and 0.361 , respectively. All these values correspond to fair agreement.

\subsubsection{Experimental Results}

In order to see the importance of each factor in a specific device, we adopt the Pearson correlation coefficients between readability factors and reading devices. Table 4 lists the four most important factors in each device and their Pearson correlation coefficients. Especially, $p$-value is smaller than 0.05 for all factors in this table.

For the smart phone, SL, the number of sentences in a text, is the most important readability factor. Its correlation with the smart phone is -0.394 . TL, the number of characters, is the second important factor and has a negative correlation of -0.293 . These results imply that readers are negatively sensitive to the length of an article because of the small display size of a smart phone. That is, in the smart phone, longer articles are recognized as difficult to read compared to shorter ones. The number of words per sentence, WS, is the third important factor with correlation of 0.288 . The log-likelihood of an article, LL, is also positively related with the readability, which proves that widely-used words make it easy to understand an article. The top three factors are superficial with regard to text length. Therefore, the superficial factors are more important than other types of factors for the smart phone.
SL is the most critical readability factor even for the tablet. It affects readability with high correlation of 0.370 . The second important factor is WS with correlation of 0.321 . Both of these factors are superfical. The third important factor, LL, is positively related with readability as expected. The fourth factor that affects readability is the number of noun phrases, NP. It is natural for NP to be positively related with the readability.

Finally, for the paper, NP is most strongly related to readability with correlation of 0.298 . The second important factor is WS, whose correlation is 0.278 . LL is the third important factor and shows a positive relationship. Note that WS and LL are important readability factors for all devices. The next important readability factor for the paper is the average number of verb phrases (VP). The articles with many noun phrases and verb phrases are perceived as easier-to-read for the paper. Note that the importance of superficial factors is limited for the paper. We expected that WS is negatively related, but, it is positively related with readability for all three devices. The reason for this could be that the annotators thought the articles with higher WS are more interesting.

The important factors for the smart phone are different from those for the paper. On the other hand, the tablet shares many factors with both the smart phone and the paper. Because the screen size of a tablet is similar to the size of an A4 paper, the tablet and the paper share readability factors. However, length-related factors play a more important role than syntactic factors in the smart phone because a smart phone has a smaller screen.

\subsection{Experiments on News Recommendation}

\subsubsection{Experiment Settings}

Experiments for news article recommendation were performed to see the effectiveness of device-dependent readability. The process of news recommendation with device-dependent readability is as follows. For a specific device,

1. Select top- $k$ news articles from a news repository by the criterion of the recommendation system.

2. Re-rank the $k$ articles by the readability of the device using Equation (3).

3. Select top- $k^{*}$ news articles by the new rank.

4. Human annotators read and rate the $k^{*}$ articles with the device.

5. Compare the ranks of $k^{*}$ articles by devicedependent readability with those by human ratings.

Since we have three types of devices, this process is performed three times with a different device.

The news articles from September 10, 2013 to September 12, 2013 collected from Naver News were 


\begin{tabular}{|c|c|c|c|}
\hline & Min & Max & Average \\
\hline Article length & 277 & 6,077 & 990.68 \\
\# of sentences & 4 & 199 & 22.85 \\
\# of words per sentence & 4 & 100 & 15.73 \\
\# of words per article & 71 & 2,034 & 301.61 \\
\hline
\end{tabular}

Table 5: Statistics of news data for recommendation

\begin{tabular}{|c|c|c|c|}
\hline Reading device & Min & Max & Average \\
\hline Smart phone & 1 & 5 & $3.513 \pm 0.962$ \\
Tablet & 1 & 5 & $3.344 \pm 0.852$ \\
Paper & 1 & 5 & $3.250 \pm 0.907$ \\
\hline
\end{tabular}

Table 6: Scores of news articles by human annotators in news recommendation

used as the news repository. The number of times that a news article was actually read by its anonymous readers at the portal site is used as the criterion for the recommendation system. Since this criterion is provided on a daily basis and news articles were collected for three days, the process explained above is performed three times. The top twenty articles were selected by the criterion every day. That is, $k=20$. Table 5 shows the statistics of the total 60 articles. The shortest article consists of 277 characters, and the longest article has 6,077 characters. On average, an article is written with 990.68 characters. The minimum number of sentences in an article is 4, and the maximum number of sentences is 199. An article is composed of 22.85 sentences on average. The average number of words in a sentence is 15.73 , whereas a sentence length ranges from 4 to 100 words. The shortest article has 71 words, and the longest article has 2,034 words. One article has approximately 301.61 words on average.

Three human annotators labeled the scores of the news articles manually. The annotators were the same persons who labeled the readability scores. Similar to the previous experiments, 7 days intervals was placed among devices to reduce the memory effect. The same two questions used in the previous section were used again for this experiment. The annotators assigned a score between 1 and 5 to every article for each question. The final score of an article was obtained by averaging six scores (two questions from three annotators). Table 6 summarizes the scores of the articles by the human annotators. As shown in this table, the article scores vary for all reading devices. The average scores for smart phone, tablet, and paper are 3.513, 3.344, and 3.250 respectively. The Kappa value for the 'smart phone' is 0.402 , and that for both the 'tablet' and the 'paper' is 0.393 . Thus, the value of 'smart phone' falls into moderate agreement, whereas those of the 'tablet' and 'paper' correspond to fair agreement. The performance of the news article recommendation is evaluated with the Normalized Discounted Cumulative Gain at top P (NDCG@P) (Järvelin and Kekäläinen, 2002).

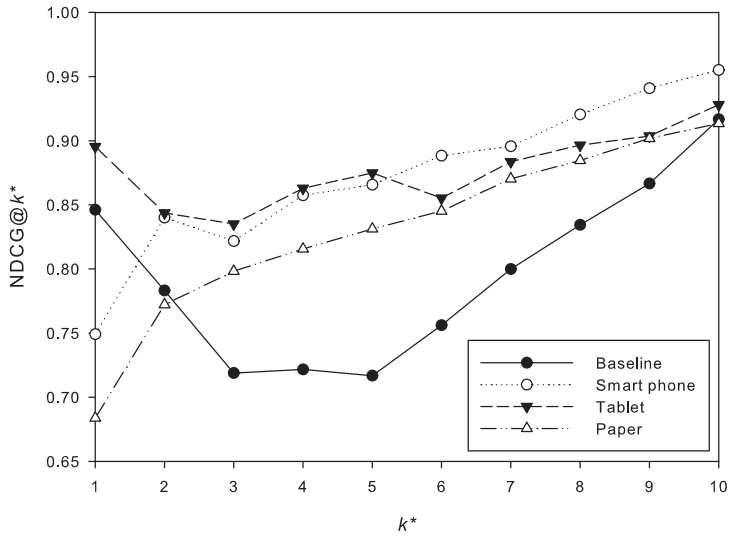

Figure 2: NDCG@ $k^{*}$ scores with various $k^{*}$ for the smart phone.

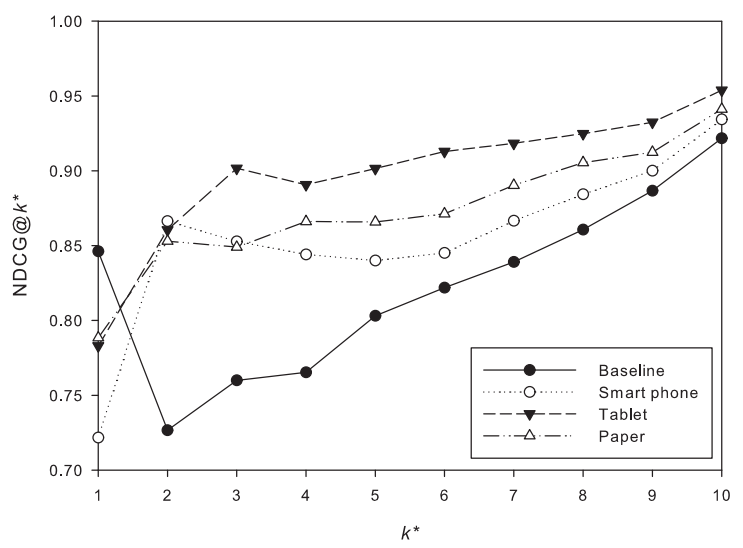

Figure 3: NDCG@ $k^{*}$ scores with various $k^{*}$ for the tablet.

\subsubsection{Experimental Results}

For the a baseline criterion, we use the news article recommendation system in Naver, which recommends news article by the number of article hits. Figures 2 to 4 show the NDCG@ $k^{*}$ scores with $1 \leq k^{*} \leq 10$ for the three devices. Each graph in these figures compares the performance of various devices when the readability for a specific device is used. That is, Figure 2 depicts the NDCG@ $k^{*}$ scores for the recommended news articles when the articles are shown in the smart phone, the tablet, and the paper respectively. In computing their NDCG@ $k^{*}$ scores, the news articles are re-ranked by readability for the smart phone. Therefore, in this figure we expect that the NDCG@ $k^{*}$ score for using the smart phone is higher than those for using the tablet and paper. In the same way, Figure 3 and Figure 4 compare the NDCG@ $k^{*}$ scores when the readabilities for the tablet and paper are used.

In all three graphs, the best news recommendation performance is achieved when the device used to read 


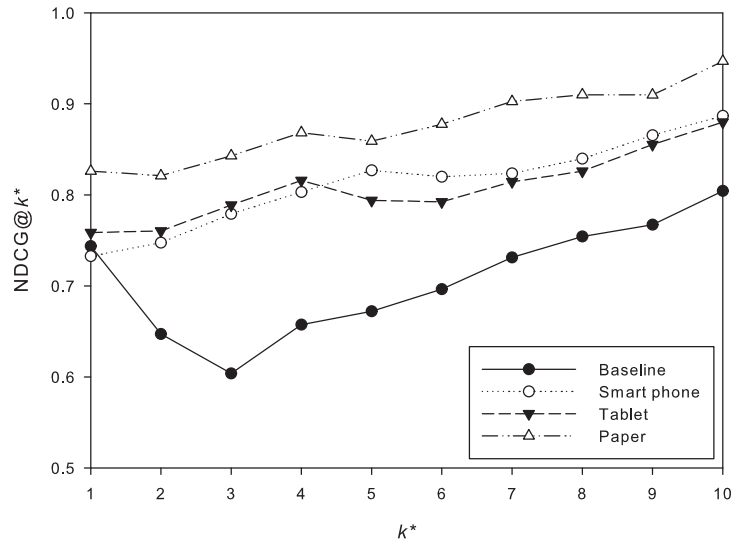

Figure 4: NDCG@ $k^{*}$ scores with various $k^{*}$ for the paper.

news articles is the same as the device used for readability. In Figure 2, the use of the smart phone outperforms those of other devices when $k^{*} \geq 6$. This proves that the quality of highly ranked news articles is much better for the smart phone than for other devices, when the readability for smart phone is used.

Figure 3 shows the NDCG@ $k^{*}$ scores for using various devices when the news articles are re-ranked by readability for the tablet. In this figure, the use of the tablet as a reading device is better than using the smart phone or the paper. The performance difference is largest at $k^{*}=3$. The difference becomes smaller as $k^{*}$ increases up to 10 , but the performance of tablet is still higher than those of others. In Figure 2 and 3, when $k^{*}=1$, the baseline outperforms other devices. We believe this happens because the baseline chooses news articles by user-hit. Therefore, many articles recommended by the baseline are interesting because people tend to click more often when an article is interesting. As noted, readability reflects users' interests, which leads to high performance of the baseline. The performance of paper is best in Figure 4, since the articles are re-ranked by the readability for paper. Paper outperforms all other devices for all $k^{*}$ s. Note that the performances of the baseline are always lowest regardless of reading device.

From all results above, we can infer that the use of device-dependent readability is helpful to news article recommendation. This is because the readability factors that affect the readers of news articles are different according to the reading device. Therefore, it is important to reflect the characteristics of a reading device when recommending news articles.

\section{Conclusion}

In this paper, we have proposed a device-dependent readability. Since a reading device is one of the most important features of readability, different weights have been assigned to the readability factors according to device type. We have shown that the important readability factors are distinct according to the reading device by investigating the correlation between the readability factors and the reading device. Through the correlation, we found that tablet shares many important factors with both smart phone and paper.

The experiments on the news articles collected from an Internet portal proved that readability is actually affected by the reading device. In addition, the validity of the device-dependent readability was shown by applying it to the news article recommendation. The news articles were first ranked by the criterion of the recommendation system. Then, they were re-ranked by the device-dependent readability. Our experiments showed that the recommendation performance of the re-ranked articles gets best when the device used for readability is the same as the reading device. These two types of experiments proved the importance and effectiveness of the device-dependent readability.

\section{Acknowledgments}

This work was supported by the IT R\&D program of MSIP/KEIT (10044494, WiseKB: Big data based selfevolving knowledge base and reasoning platform) and the Industrial Strategic Technology Development Program (10035348, Development of a Cognitive Planning and Learning Model for Mobile Platforms) funded by the Ministry of Knowledge Economy(MKE, Korea).

\section{References}

Alan Bailin and Ann Grafstein. 2001. The linguistic assumptions underlying readability formulae: A critique. Language \& Communication, 21(3):285-301.

Regina Barzilay and Mirella Lapata. 2008. Modeling local coherence: An entity-based approach. Сотриtational Linguistics, 34(1):1-34.

Edgar Dale and Jeanne Chall. 1949. The concept of readability. Elementary English, 26(1):19-26.

Abhinandan Das, Mayur Datar, Ashutosh Garg, and Shyam Rajaram. 2007. Google news personalization: scalable online collaborative filtering. In Proceedings of the 16th International Conference on World Wide Web, pages 271-280.

Joseph Fleiss. 1971. Measuring nominal scale agreement among many raters. Psychological bulletin, 76(5):378-382.

Rudolph Flesch. 1948. A new readability yardstick. Journal of Applied Psychology, 32(3):221-233.

Thomas François and Cédrick Fairon. 2012. An AI readability formula for French as a foreign language. In Proceedings of the 2012 Joint Conference on Empirical Methods in Natural Language Processing and Computational Natural Language Learning, pages 466-477. 
Michael Halliday and Ruqaiya Hasan. 1976. Cohesion in English. Longman Group Ltd.

Satoshi Hasegawa, Kazuhiro Fujikake, Masako Omori, and Masaru Miyao. 2008. Readability of characters on mobile phone liquid crystal displays. International Journal of Occupational Safety and Ergonomics (JOSE), 14(3):293-304.

Michael Heilman, Kevyn Collins-Thompson, and Maxine Eskenazi. 2008. An analysis of statistical models and features for reading difficulty prediction. In Proceedings of the Third Workshop on Innovative Use of NLP for Building Educational Applications, pages 71-79.

Kristina Hillbom. 2009. Newspaper Readability: a Broadsheet vs. a Tabloid. Ph.D. thesis, University of Gävle.

Måns Huldén. 2004. Linguistic complexity in two major american newspapers and the associated press newswire, 1900-2000. Master's thesis, Åbo Akademi University.

Shoaib Jameel, Wai Lam, and Xiaojun Qian. 2012. Ranking text documents based on conceptual difficulty using term embedding and sequential discourse cohesion. In Proceedings of the The 2012 IEEE/WIC/ACM International Joint Conferences on Web Intelligence and Intelligent Agent TechnologyVolume 01, pages 145-152.

Kalervo Järvelin and Jaana Kekäläinen. 2002. Cumulated gain-based evaluation of IR techniques. ACM Transactions on Information Systems (TOIS), 20(4):422-446.

J. Peter Kincaid, Robert Fishburne Jr., Richard Rogers, and Brad Chissom. 1975. Derivation of new readability formulas (automated readability index, fog count and flesch reading ease formula) for navy enlisted personnel. Technical report, DTIC Document.

Harry Kitson. 1927. The mind of the buyer. MacMillan Company.

Lihong Li, Wei Chu, John Langford, and Robert E. Schapire. 2010. A contextual-bandit approach to personalized news article recommendation. In Proceedings of the 19th International Conference on World Wide Web, pages 661-670.

Jiahui Liu, Peter Dolan, and Elin R. Pedersen. 2010. Personalized news recommendation based on click behavior. In Proceedings of the 15th International Conference on Intelligent User Interfaces, pages 3140 .

Yi Ma, Eric Fosler-Lussier, and Robert Lofthus. 2012. Ranking-based readability assessment for early primary children's literature. In Proceedings of the 2012 Conference of the North American Chapter of the Association for Computational Linguistics: $\mathrm{Hu}$ man Language Technologies, pages 548-552.
Naoaki Okazaki, Yutaka Matsuo, and Mitsuru Ishizuka. 2005. Improving chronological ordering of sentences extracted from multiple newspaper articles. ACM Transactions on Asian Language Information Processing (TALIP), 4(3):321-339.

Gustav Öquist. 2006. Evaluating readability on mobile devices. Ph.D. thesis, Uppsala University.

Emily Pitler and Ani Nenkova. 2008. Revisiting readability: A unified framework for predicting text quality. In Proceedings of the Conference on Empirical Methods in Natural Language Processing, pages 186-195.

Jack Richards, John Platt, Heidi Platt, and Christophe Candlin. 1992. Longman Dictionary of Language Teaching and Applied Linguistics, volume 78. Longman London.

Sarah Schwarm and Mari Ostendorf. 2005. Reading level assessment using support vector machines and statistical language models. In Proceedings of the 43rd Annual Meeting on Association for Computational Linguistics, pages 523-530.

Xin Yan, Dawei Song, and Xue Li. 2006. Conceptbased document readability in domain specific information retrieval. In Proceedings of the 15th ACM International Conference on Information and Knowledge Management, pages 540-549.

Mostafa Zamanian and Pooneh Heydari. 2012. Readability of texts: State of the art. Theory and Practice in Language Studies, 2(1):43-53. 